\title{
Spectral analysis of boundary layers in Rayleigh-Bénard convection
}

\author{
Jos Verdoold, ${ }^{1, *}$ Maarten van Reeuwijk, ${ }^{2}$ Mark J. Tummers, ${ }^{1}$ Harm J. J. Jonker, ${ }^{1}$ and Kemo Hanjalić ${ }^{1,3}$ \\ ${ }^{1}$ Department of Multi-Scale Physics, Delft University of Technology, Lorentzweg 1, 2628 CJ Delft, The Netherlands \\ ${ }^{2}$ Department of Civil and Environmental Engineering, Imperial College London, London SW7 2AZ, United Kingdom \\ ${ }^{3}$ Department of Mechanics and Aeronautics, University of Rome, "La Sapienza," Rome, Italy
}

(Received 17 July 2007; revised manuscript received 12 October 2007; published 9 January 2008)

\begin{abstract}
A combined experimental and numerical study of the boundary layer in a 4:1 aspect-ratio Rayleigh-Bénard cell over a four-decade range of Rayleigh numbers has been undertaken aimed at gaining a better insight into the character of the boundary layers. The experiments involved the simultaneous laser Doppler anemometry measurements of fluid velocity at two locations, i.e., in the boundary layer and far away from it in the bulk, for Rayleigh numbers varying between $1.6 \times 10^{7}$ and $2.4 \times 10^{9}$. In parallel, direct numerical simulations have been performed for the same configuration for Rayleigh numbers between $7.0 \times 10^{4}$ and $7.7 \times 10^{7}$. The temperature and velocity probability density functions and the power spectra of the horizontal velocity fluctuations measured in the boundary layer and in the bulk flow are found to be practically identical. Except for the smallest Rayleigh numbers, the spectra in the boundary layer and in the bulk central region are continuous and have a wide range of active scales. This indicates that both the bulk and the boundary layers are turbulent in the Ra number range considered. However, molecular effects can still be observed and the boundary layer does not behave like a classical shear-driven turbulent boundary layer.
\end{abstract}

DOI: 10.1103/PhysRevE.77.016303

PACS number(s): 47.20.Bp, 47.27.nb, 44.25.+f

\section{INTRODUCTION}

Rayleigh-Bénard (RB) convection in a fluid trapped between two horizontal plates of unequal temperature with the bottom wall being warmer than the top wall has long served as a paradigm of thermal convection. Despite numerous studies (see, e.g., overviews by $[1,2]$ ), it continues to pose challenges because of a number of still unresolved and controversial issues. We recall that $\mathrm{RB}$ convection is characterized by the Rayleigh number $\mathrm{Ra}=\alpha g \Delta \Theta H^{3} /(\nu \kappa)$ and the Prandtl number $\operatorname{Pr}=\nu / \kappa$, where $\alpha$ is the thermal expansion coefficient, $\Delta \Theta$ is the temperature difference between the cold and the hot plate, $H$ is the vertical distance between the plates, $\nu$ is the kinematic viscosity, and $\kappa$ is the thermal diffusivity. In practical RB setups the lateral size $W$ of the plates is finite. This is taken into account by a third dimensionless number known as the aspect ratio $\Gamma=W / H$.

A topic of intense debate is the scaling law for the dimensionless heat transfer, $\mathrm{Nu} \sim \mathrm{Ra}^{\gamma}$, especially for the range $\mathrm{Ra}>10^{7}$ [3]. Here $\mathrm{Nu}$ is the Nusselt number $\mathrm{Nu}=h H / \lambda$ $=-H /(\Delta \Theta) \partial \bar{\Theta} /\left.\partial z\right|_{\text {wall }}, h$ stands for the convective heat transfer coefficient, $\lambda$ is the thermal conductivity, and $\bar{\Theta}$ is the averaged temperature. References [4-6] proposed scaling laws for the heat transfer and temperature statistics in RB convection, which differed from the classical theories, such as that of [7]. In the search for general scaling behavior, Ref. [8] presented a unifying theory dividing the Ra-Pr plane into several regions, each with different scaling. Crucial assumptions in this theory are the existence of a large-scale circulation, also known as the wind, and a Blasius type laminar boundary layer. The assumption that the boundary layers of Rayleigh-Bénard convection behave as a laminar Blasius boundary layer is supported by several observations: (i) the

\footnotetext{
*j.verdoold@tudelft.nl
}

scaling of the friction factor $C_{f}$ is compatible with that of a laminar boundary layer past a flat plate at moderate $\mathrm{Ra}$ [9-11]; (ii) the typical Reynolds number Re of the flow is low at moderate $\mathrm{Ra}$ : for water $\mathrm{Re} \approx 200$ at $\mathrm{Ra}=10^{7}$ and $\Gamma=4$, which is generally accepted to be too low to sustain turbulence. Reference [8] argued that at large enough Ra this laminar boundary layer breaks down and becomes turbulent, presuming that the transition to a turbulent boundary layer is shear triggered. The critical Ra number for this transition was estimated to be in the order of $10^{14}$ for $\operatorname{Pr}=1$.

Despite this apparently convincing evidence, it is not clear how a boundary layer which is highly unsteady due to continuous plume impingement and detachment can behave quantitatively as a laminar boundary layer. This would indicate that the plumes only cause unsteady disturbances and a consequent time dependence in the boundary layer but are passive otherwise.

The aim of this paper is to enhance the understanding of the dynamics of the boundary layers by comparing velocity statistics in the boundary layer with those determined in the bulk. It has widely been regarded that the main prerequisite for unsteady velocity fluctuations to be qualified as turbulence, in contrast to other uncorrelated, chaotic and random, fluctuations, is a power spectrum that is continuous over a wide range of scales. For this reason we focused on the analysis of spectral density functions (SDFs), complemented with the probability density functions (PDFs), aimed at clarifying the nature of the fluctuations in the boundary layer. For this purpose we use in parallel complementary numerical and experimental techniques: direct numerical simulations (DNSs) are performed for the lower Ra values, laser Doppler anemometry (LDA) measurements are conducted for the medium and higher Ra values. All experiments and simulations combined cover a $\mathrm{Ra}$ range between $7.0 \times 10^{4}$ and $2.4 \times 10^{9}$. In the midrange both techniques overlap over about one decade. 


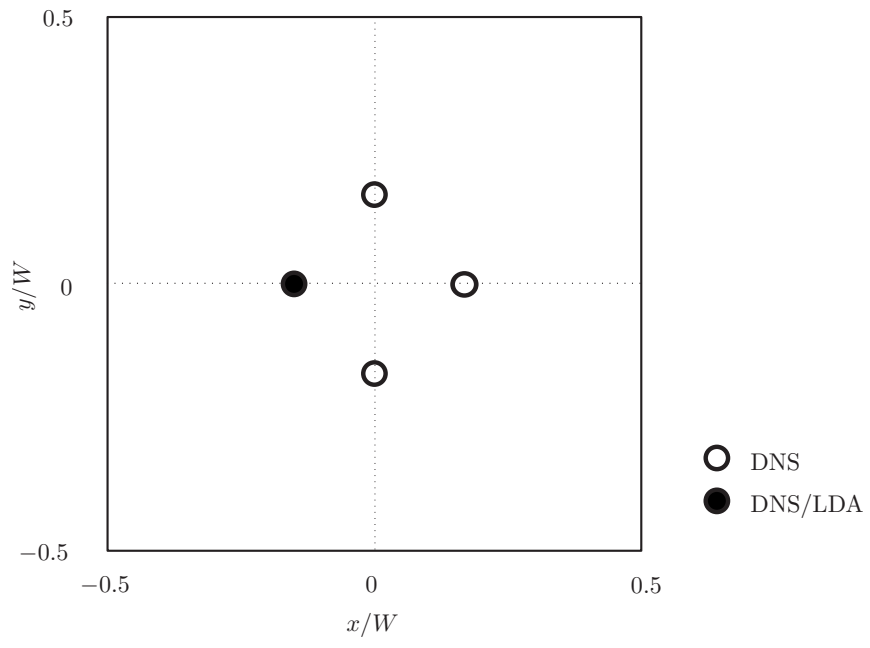

FIG. 1. Schematic of the measurement points in the RB cell for both DNS and LDA measurements.

\section{METHODOLOGY}

\section{A. Experiments}

The experiments were conducted in a $600 \times 600$ $\times 155 \mathrm{~mm}^{3}(W \times W \times H)$ cell filled with water (described in more detail in [12]). In brief, two copper plates at the top and bottom are kept at a constant temperature by passing water through the plates' internal channels, drawn from two basins containing constant temperature water (inaccuracy less than $0.03 \mathrm{~K})$. The plates thus impose a controlled temperature difference $\Delta \Theta$ on the working fluid. Throughout this paper we will use a Cartesian coordinate system with its origin at the center of the bottom wall, Fig. 1. The $x$ and $y$ coordinates are measured along the bottom wall, parallel to the side walls of the RB cell. The $z$ coordinate measures the wall normal distance.

Velocities were measured by using two one-component laser Doppler anemometers manufactured by Dantec. The green $(514.5 \mathrm{~nm})$ and blue $(488.0 \mathrm{~nm})$ colors of a $5 \mathrm{~W}$ argonion laser are used to simultaneously measure the horizontal component in the $x$ direction in the bulk and in the boundary layer. The measured velocity component is in the direction of the large-scale circulation. To enable the detection of instantaneous flow reversals one beam of each color was frequency pre-shifted by a Bragg cell. The light scattered by $5 \mu \mathrm{m}$ polyamid seed particles was collected in the on-axis forward direction, which, in comparison to backscattering, results in a superior signal-to-noise ratio of the Doppler signals. The photomultiplier output signals were first downmixed electronically to an effective preshift of $30 \mathrm{kHz}$ and then fed to two Dantec BSA signals processors to determine the instantaneous velocities. The LDA system measured the instantaneous velocities of the seed particles with an inaccuracy of $0.5 \%$. The length and diameter of both measurement volumes are 0.8 and $0.1 \mathrm{~mm}$, respectively, which is sufficiently small to resolve the smallest length scales of the flow. For each Ra number, the measurement time $T$ was at least $20 \mathrm{~h}$ to have sufficient data for an accurate statistical description of the flow.
TABLE I. Experimental details for the LDA measurements. All experiments have been performed in a $\Gamma=4$ aspect ratio domain. $T$ denotes the measurement time.

\begin{tabular}{ccccccc}
\hline \hline $\mathrm{Ra}$ & $\operatorname{Pr}$ & $\Delta \Theta[\mathrm{K}]$ & $\bar{\Theta}\left[{ }^{\circ} \mathrm{C}\right]$ & $\alpha\left[\mathrm{K}^{-1}\right]$ & $\nu\left[\mathrm{m}^{2} \mathrm{~s}^{-1}\right]$ & $T[\mathrm{~s}]$ \\
\hline $1.6 \times 10^{7}$ & 9.4 & 0.92 & 10.2 & $8.8 \times 10^{-5}$ & $1.3 \times 10^{-6}$ & $1.1 \times 10^{5}$ \\
$8.2 \times 10^{7}$ & 7.0 & 1.57 & 19.9 & $2.1 \times 10^{-4}$ & $1.0 \times 10^{-6}$ & $0.9 \times 10^{5}$ \\
$4.2 \times 10^{8}$ & 5.5 & 6.52 & 30.0 & $3.0 \times 10^{-4}$ & $8.0 \times 10^{-7}$ & $1.2 \times 10^{5}$ \\
$2.4 \times 10^{9}$ & 5.5 & 25.1 & 30.3 & $3.0 \times 10^{-4}$ & $8.0 \times 10^{-7}$ & $1.3 \times 10^{5}$ \\
\hline \hline
\end{tabular}

The mean data rate varied between 10 and $30 \mathrm{~Hz}$, depending on $\mathrm{Ra}$ and distance from the wall. For moderate Ra numbers, the relatively low characteristic velocities result in a relatively low data rate. Measurement locations close to the wall have an inherently low data rate due to the low fluid volume flux.

Table I shows the experimental parameters as well as the relevant nondimensional numbers. The Rayleigh number is varied by changing the temperature difference $\Delta \Theta$ between the bottom and top plates from nearly $1 \mathrm{~K}$ at $\mathrm{Ra}=1.6 \times 10^{7}$ to $25 \mathrm{~K}$ at $\mathrm{Ra}=2.4 \times 10^{9}$. For the highest Ra numbers, the relatively large temperature differences cause larger density differences, but these remain small enough to neglect nonBoussinesq effects. The Prandtl number will change as well, but the ratio between the smallest and largest Pr number is only 1.7 .

\section{B. Simulations}

The direct numerical simulations cover the lower to moderate $\mathrm{Ra}$ number range between $\mathrm{Ra}=7.0 \times 10^{4}$ and $\mathrm{Ra}=7.7 \times 10^{7}$. The numerics are based on staggered finite differences, with a central second order scheme for advection, and a second order Adams-Bashforth time integration scheme. To effectively resolve the boundary layers, grid stretching in the wall-normal direction has been implemented, and special care has been taken to preserve the skew symmetry of the advective operator [13]. The code, as reported in [14], has been extended to support sidewalls. In the situation without sidewalls, the Poisson equation is Fouriertransformed in the two homogeneous directions. As a result, the Poisson equation decouples for these directions, and the pressure amplitudes can be solved per component by solving a tridiagonal system in the wall-normal direction. To incorporate sidewalls, the Fourier-transform was replaced by a cosine transform, which automatically satisfies a Neumann boundary condition for pressure. The sidewall boundary conditions for the velocity and temperature could be enforced directly due to the explicit time-integration.

Dirichlet boundary conditions are applied at the top and bottom plate, i.e., no-slip and isothermal boundary conditions for velocity and temperature, respectively. On the sidewalls, Neumann boundary conditions are applied for both temperature and velocity, i.e., free-slip and insulating walls for velocity and temperature. The free-slip (stress free) velocity boundary conditions were used to avoid having to resolve the boundary layer at the sidewalls. Although this changes the flow physics near the sidewalls, its global effect 
TABLE II. Simulation details for DNS.

\begin{tabular}{ccccc}
\hline \hline $\mathrm{Ra}$ & Grid & $\Delta t / t^{*}$ & $T / t^{*}$ & $\mathrm{Nu}$ \\
\hline $7.0 \times 10^{4}$ & $64^{2} \times 48$ & $4.0 \times 10^{-4}$ & 240 & 3.8 \\
$5.6 \times 10^{5}$ & $96^{2} \times 64$ & $2.3 \times 10^{-4}$ & 140 & 7.2 \\
$2.6 \times 10^{6}$ & $128^{2} \times 92$ & $1.7 \times 10^{-4}$ & 165 & 11.3 \\
$7.2 \times 10^{6}$ & $192^{2} \times 128$ & $1.3 \times 10^{-4}$ & 155 & 15.2 \\
$2.1 \times 10^{7}$ & $384^{2} \times 160$ & $1.0 \times 10^{-4}$ & 40 & 20.0 \\
$7.7 \times 10^{7}$ & $384^{2} \times 160$ & $1.0 \times 10^{-4}$ & 30 & 30.0 \\
\hline \hline
\end{tabular}

is limited for large aspect ratio domains, as preliminary simulations showed. Neumann boundary conditions were applied for pressure at all boundaries.

In the simulations, the properties of water are used except that $\operatorname{Pr}=7$ is kept fixed for all $\mathrm{Ra}$. The grid resolution (Table II) is sufficient to resolve the smallest turbulent scales, i.e., the Kolmogorov scale $\eta_{\mathrm{K}}=\left(\nu^{3} / \varepsilon\right)^{1 / 4}$ and the Corrsin scale $\eta_{\mathrm{C}}=\operatorname{Pr}^{-1 / 2} \eta_{\mathrm{K}}$. At $\mathrm{Pr}=7$, the Corrsin scale dominates the grid requirements, and the resolution roughly corresponds to $\Delta x \approx \Delta y \approx \Delta z \approx 4 \eta_{\mathrm{C}}$. To resolve the boundary layers at the top and bottom wall properly, grid stretching has been applied in the wall-normal direction, such that at least eight points are within the thermal boundary layer. It should be noted that the simulation at the highest $\mathrm{Ra}$ number $\left(=7.7 \times 10^{7}\right)$ may be slightly underresolved in the horizontal directions. High quality simulation as reported in [15] at $\mathrm{Ra}=10^{8}$ and $\operatorname{Pr}=7$ required 768 points per horizontal direction, but reduction to 384 points was the only way to simulate over a sufficiently long time interval to obtain reliable one-point statistics required for the comparison with the experiments. The additional information shown in Table II are the dimensionless time step $t / t^{*}$, the number of simulated turnovers $T / t^{*}$, and the average Nusselt number $\mathrm{Nu}$. The typical convective turnover time $t^{*} \equiv H / U$ with $U$ the typical velocity based on the free fall scaling. The average Nusselt number is calculated as $\mathrm{Nu}=-H /(\Delta \Theta) \partial \bar{\Theta} /\left.\partial z\right|_{\text {wall }}$, with $\bar{\Theta}$ averaged over the entire bottom surface and over many turnovers.

\section{Measurement locations}

Simultaneous measurements have been performed in the boundary layer and in the bulk of the cell for various Ra. The measurement location in the bulk is at $x_{\text {bulk }}=W / 3$, $y_{\text {bulk }}=W / 2$ with a fixed wall distance $z_{\text {bulk }}=H / 2$; see Fig. 1 . The measurement location in the boundary layer has the same $x$ and $y$ coordinates, but the wall distance is different, i.e., $z_{\mathrm{bl}}=0.4 \lambda_{u}$, where $\lambda_{u}$ is the kinematic boundary layer thickness determined as in [8]:

$$
\lambda_{u}=H / \sqrt{\mathrm{Re}} .
$$

In this expression for $\lambda_{u}$ the Reynolds number Re was taken from $[8,16]$ :

$$
\operatorname{Re}=0.073 \operatorname{Ra}^{1 / 2} \operatorname{Pr}^{-5 / 6} .
$$

Table III lists the physical measurement locations, where
TABLE III. Measurement heights for the different Ra experiments. The Re number is based on Eq. (2).

\begin{tabular}{cccccc}
\hline \hline & $\mathrm{Ra}$ & $\mathrm{Pr}$ & $\mathrm{Re}$ & $\lambda_{u} / H$ & $z_{\mathrm{bl}} / H$ \\
\hline \multirow{2}{*}{ DNS } & $7.0 \times 10^{4}$ & 7.0 & 3.8 & 0.512 & 0.204 \\
& $5.6 \times 10^{5}$ & 7.0 & 11 & 0.305 & 0.121 \\
& $2.6 \times 10^{6}$ & 7.0 & 23 & 0.207 & 0.0827 \\
& $7.2 \times 10^{6}$ & 7.0 & 39 & 0.161 & 0.0641 \\
& $2.1 \times 10^{7}$ & 7.0 & 66 & 0.123 & 0.0490 \\
& $7.7 \times 10^{7}$ & 7.0 & $1.3 \times 10^{2}$ & 0.0884 & 0.0354 \\
LDA & $1.6 \times 10^{7}$ & 9.4 & 48 & 0.145 & 0.0578 \\
& $8.2 \times 10^{7}$ & 7.0 & $1.2 \times 10^{2}$ & 0.0897 & 0.0357 \\
& $4.2 \times 10^{8}$ & 5.5 & $4.2 \times 10^{2}$ & 0.0485 & 0.0193 \\
& $2.4 \times 10^{9}$ & 5.5 & $1.0 \times 10^{3}$ & 0.0309 & 0.0123 \\
\hline \hline
\end{tabular}

the DNS values have been rescaled to $H=155 \mathrm{~mm}$. Figure 2 shows that the wall distance for the boundary layer measurements slightly deviates from linear behavior (on a double-log plot) due to minor changes in Pr for the experimental points. In separate experiments, LDA was used to determine mean-velocity and rms-velocity profiles above the wall (at $\mathrm{Ra} \approx 8.0 \times 10^{8}$ ). The results of these experiments confirmed that the measurement locations at $z=z_{\mathrm{bl}}$ were all located in the boundary layer for the full Ra number range considered in this study. The DNS values for $\lambda_{u}$, denoted by solid circles in Fig. 2, support this observation. Clearly, Eq. (1) overestimates the scaling exponent of $\lambda_{u}$, so that the prefactor $\alpha$ in the BL measurement points $z_{\mathrm{bl}}=\alpha \lambda_{u}$ is a decreasing function of Ra. Hence, as $\mathrm{Ra}$ increases, the measurements are performed deeper into the kinematic boundary layer. The disparity between the theoretical prediction (1) and the measurements for $\lambda_{u}$ will be discussed in more detail at the end of Sec. III B.

The DNS time series are short in respect to the LDA series. To enable a comparison between LDA and DNS results, the DNS data are extracted from four points in the

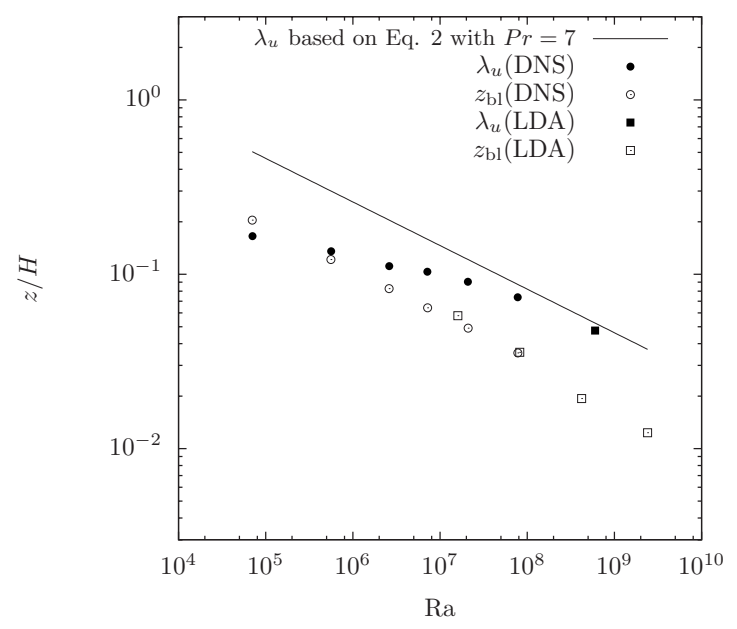

FIG. 2. Kinematic boundary-layer height $\lambda_{u}$, based on Eq. (2) as well as from DNS, and the measurement locations $z_{\mathrm{bl}}$ as function of Ra. 


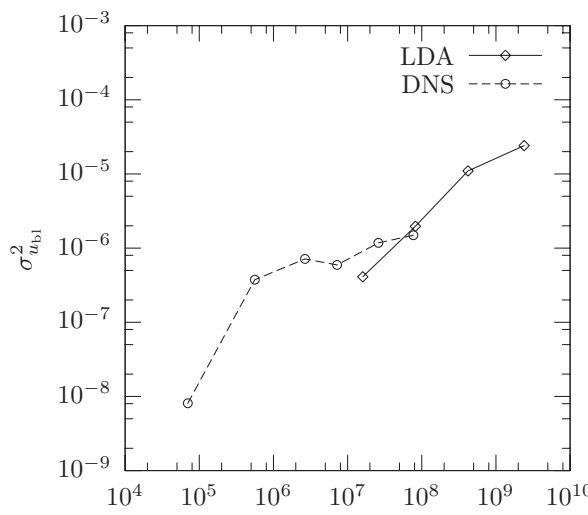

(a)

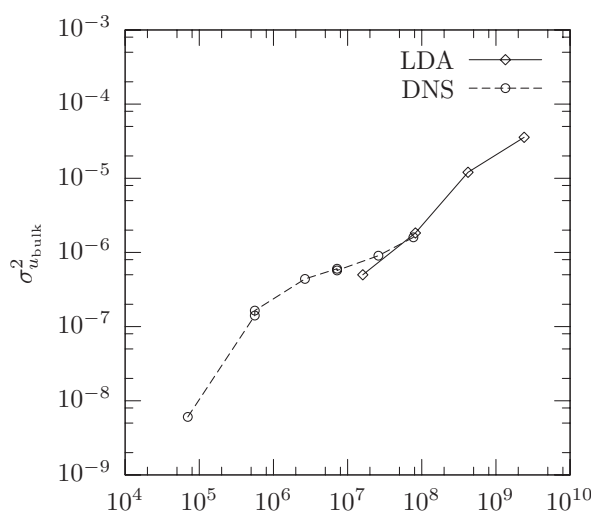

(b)

Ra

FIG. 3. Variances of the horizontal velocity component $u$ in boundary layer (a) and in the bulk (b).

domain. These points are symmetrically placed around the origin with horizontal coordinates $( \pm W / 6,0),(0, \pm W / 6)$, as shown in Fig. 1. The four obtained time series are regarded as nearly statistically independent, and are then averaged to effectively improve the statistics of the DNS time series.

\section{RESULTS}

\section{A. Probability density functions}

Figure 3 shows the measured and computed variance of the horizontal velocity $\sigma_{u}^{2}$ in the boundary layer and in the bulk. The graph shows that for increasing $\mathrm{Ra}$ there is a gradual transition of the variances between DNS and LDA results giving confidence in the procedure followed.

Temperature PDFs obtained from DNSs for the boundary layer and the bulk are depicted in Fig. 4(b) and show an exponential distribution even for $\mathrm{Ra}=5.6 \times 10^{5}$. The straight lines (on a logarithmic scale) are generally associated with the hard turbulent regime, which sets in at $\mathrm{Ra}=4 \times 10^{7}$ for aspect-ratio- 1 cells (e.g., $[4,5,17]$ ). Recall that the transition between soft and hard turbulence takes place at relatively low $\mathrm{Ra}$ number in the aspect-ratio-4 cell; see [18]. The boundary layer PDF is skewed toward positive temperatures. This is generally attributed to the interaction of thermal plumes with the flow. Plumes leaving the boundary layer result in positive temperature excursions.

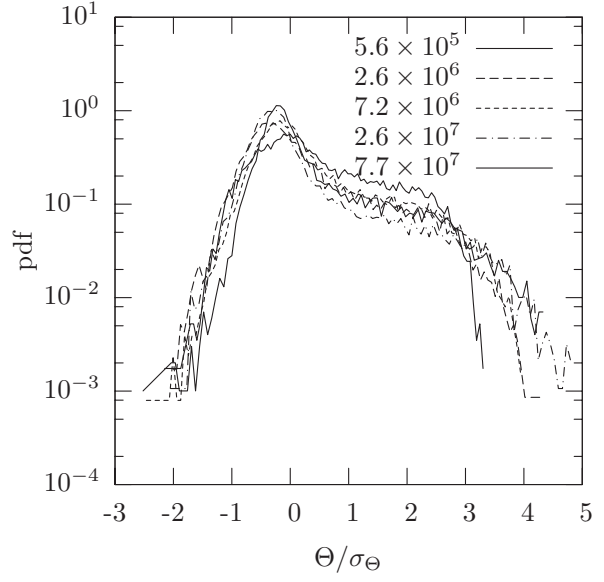

(a) boundary layer

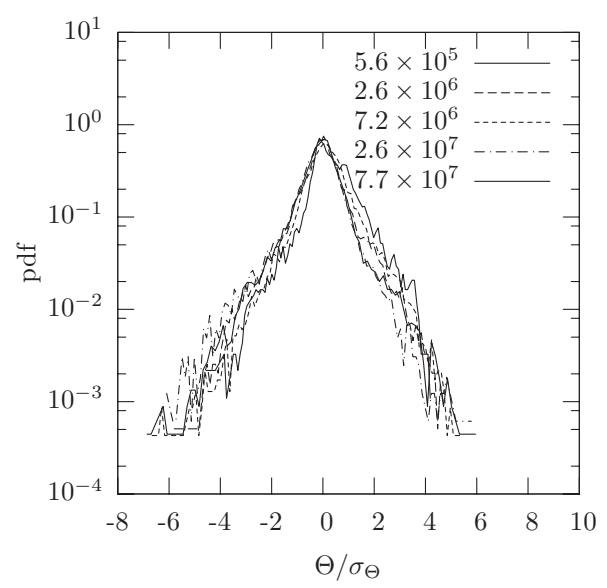

(b) bulk

FIG. 4. Probability density functions for temperature $\Theta$ in the boundary layer (a) and in the bulk (b) of the RB cell obtained from DNS. Histograms are scaled with their rms value $\sigma_{\Theta}$.

Figure 5 depicts the probability density functions (PDFs) of the computed and measured $u$ component. As expected, the PDFs of both horizontal velocity components were found to have the same shape in the simulations. For brevity only the $u$ component is therefore depicted in Fig. 5. For a better comparison, the PDFs are scaled with the rms value $\sigma_{u}$. The results show that the PDFs in both the boundary layer and in the bulk exhibit a nearly Gaussian-like shape for the complete $\mathrm{Ra}$ number range. Only the PDFs at $\mathrm{Ra}=7 \times 10^{4}$ deviate largely from this trend, mainly due to the presence of a strong oscillatory component in the velocity time series, as will be shown later when discussing the power spectra.

References $[19,20]$ make a distinction between subGaussian, Gaussian, and hyper-Gaussian stages in the development of turbulence. They argue that the nearly Gaussian shape of the PDFs in the present study indicates that turbulence is in a fully developed stage. The PDFs for the highest $\mathrm{Ra}$ values have nonzero mean values. This is caused by the large-scale circulation or "wind" within the RB cell. For $\mathrm{Ra}=4.2 \times 10^{8}$ the mean value is approximately $5 \mathrm{~mm} \mathrm{~s}^{-1}$. The wind is rather weak in the sense that its mean value is small compared to the magnitude of the rms velocity fluc- 


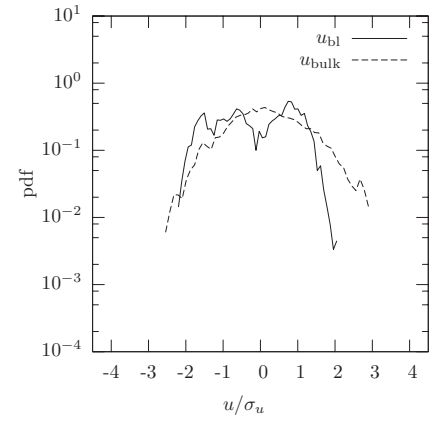

(a) $\mathrm{Ra}=7 \times 10^{4}(\mathrm{DNS})$

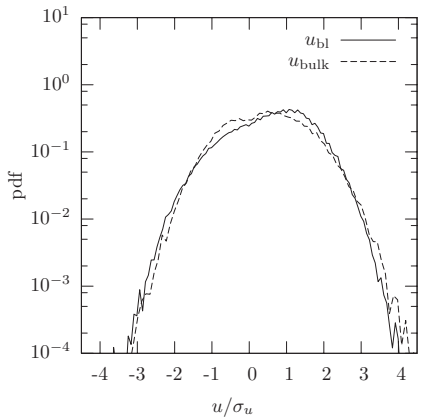

(e) $\operatorname{Ra}=1.6 \times 10^{7}(\mathrm{LDA})$

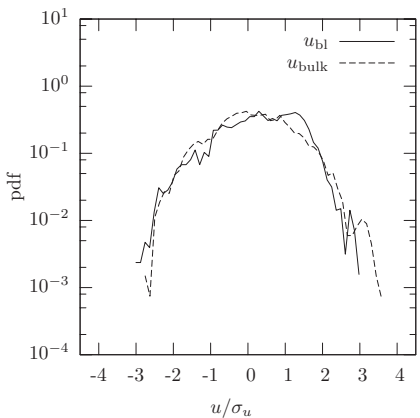

(b) $\mathrm{Ra}=5.6 \times 10^{5}(\mathrm{DNS})$

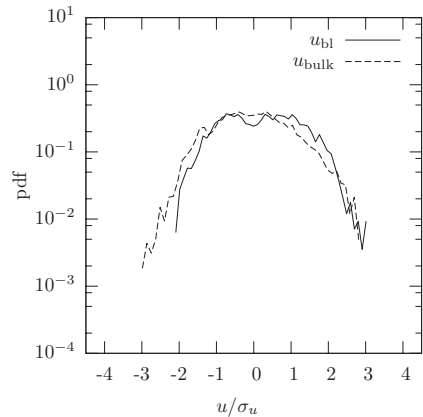

(f) $\mathrm{Ra}=2.6 \times 10^{7}$ (DNS)

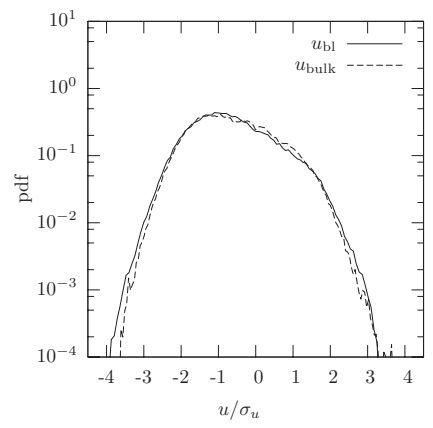

(i) $\mathrm{Ra}=4.2 \times 10^{8}(\mathrm{LDA})$

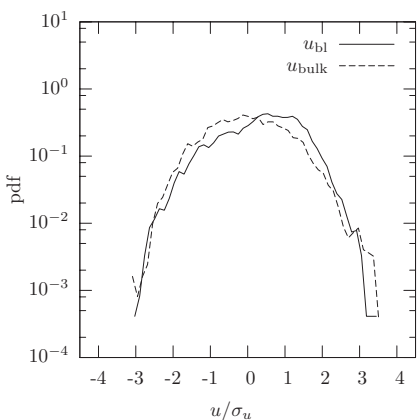

(c) $\mathrm{Ra}=2.6 \times 10^{6}(\mathrm{DNS})$

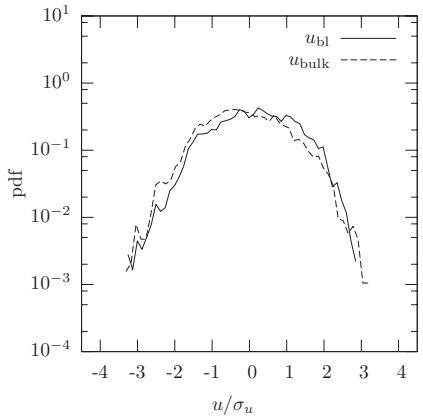

(g) $\mathrm{Ra}=7.7 \times 10^{7}$ (DNS)

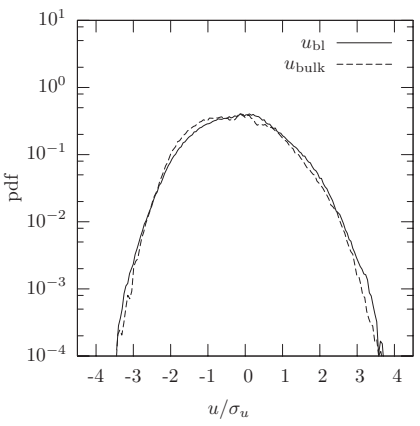

(j) $\mathrm{Ra}=2.4 \times 10^{9}(\mathrm{LDA})$

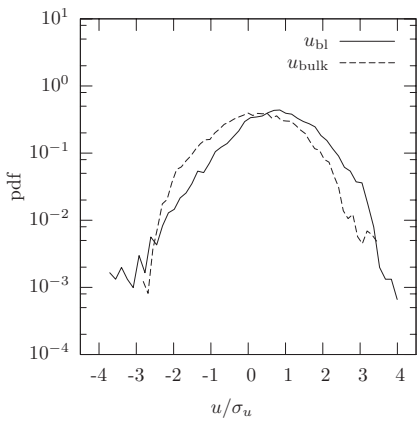

(d) $\mathrm{Ra}=7.2 \times 10^{6}$ (DNS)

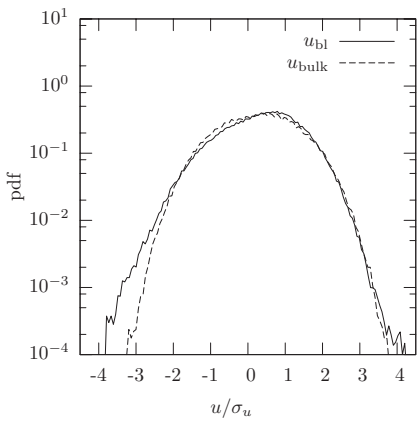

(h) $\mathrm{Ra}=8.2 \times 10^{7}(\mathrm{LDA})$

FIG. 5. Probability density functions of the $u$ horizontal velocity component in the boundary layer (bl) and in the bulk of the RB cell. Histograms are scaled with their rms value $\sigma_{u}$. The source of the data, DNS or LDA, is indicated.

tuations, in accordance with other findings reported in $[12,21-23]$. It is also observed that the wind is not always in the same direction. In some experiments [Fig. 5(h)] it appears to be clockwise, while in others [Figs. 5(i) and 5(j)] it is counterclockwise.

\section{B. Spectral density functions}

The obtained velocity time series are investigated further by computing the spectral density function (SDF). The SDF is defined as the Fourier transform of the autocorrelation function $\rho(\tau)=\overline{u^{\prime}(t) u^{\prime}(t+\tau)} / \overline{u^{\prime 2}}$, where $u^{\prime}(t)[\equiv u(t)-\bar{u}]$ is the fluctuating component of the turbulent velocity, $t$ is time, $\tau$ is the time lag, and the overline denotes ensemble averaging. All SDFs are calculated using the same algorithm, to make a fair comparison for both DNS and LDA data. This algorithm, described in [24], can account for the randomness of the sampling times in LDA measurements.
Figure 6 shows the SDFs of the horizontal component in the $x$ direction determined in the boundary layer and in the bulk for the full range of Ra considered in this study. The SDFs determined from DNS data are accurate at high frequencies, but less reliable in the low frequency range due to the relatively small measurement times. In contrast, the low frequency region in the LDA SDFs is accurate, while the high frequency range suffers from a relatively high statistical scatter caused by the random sampling process in LDA. The level of the statistical scatter (which strongly depends on the mean data rate) sets a lower limit to the spectral density that can be accurately determined from LDA data.

When comparing the various SDFs in Fig. 6 it is clear that the SDF for $\mathrm{Ra}=7 \times 10^{4}$ is different from all others. At this low Ra number, the flow is not turbulent but in a state of spatiotemporal chaos. As mentioned earlier, the velocity time series is characterized by strong oscillations. As a consequence, the SDF in Fig. 6(a) [and to a lesser extent the SDF 


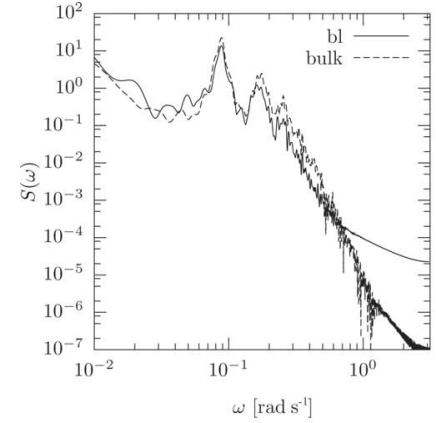

(a) $\mathrm{Ra}=7 \times 10^{6}$ (DNS)

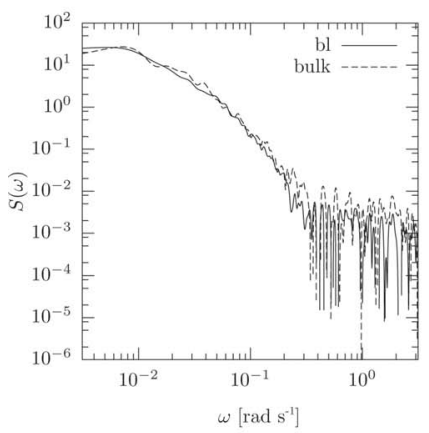

(e) $\mathrm{Ra}=1.6 \times 10^{7}$ (LDA)

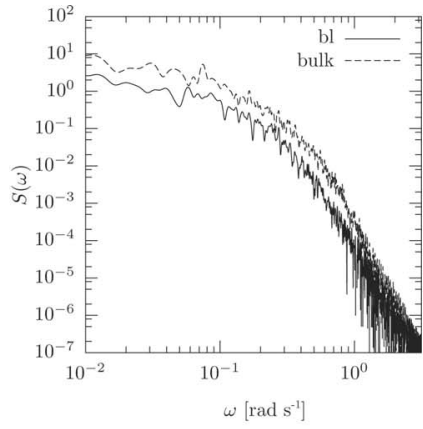

(b) $\mathrm{Ra}=5.6 \times 10^{5}$ (DNS)

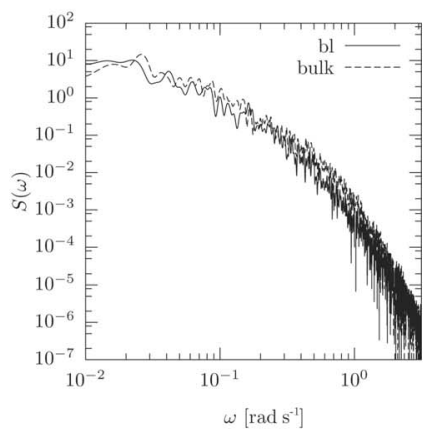

(f) $\mathrm{Ra}=2.6 \times 10^{7}$ (DNS)

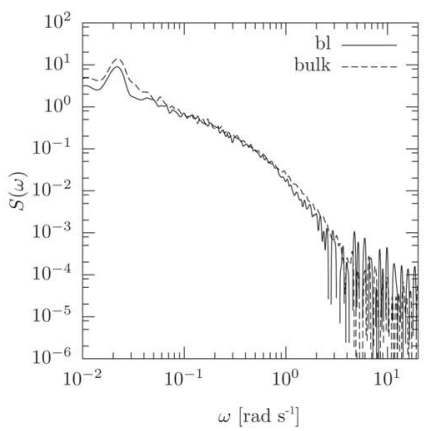

(i) $\mathrm{Ra}=4.2 \times 10^{8}$ (LDA)

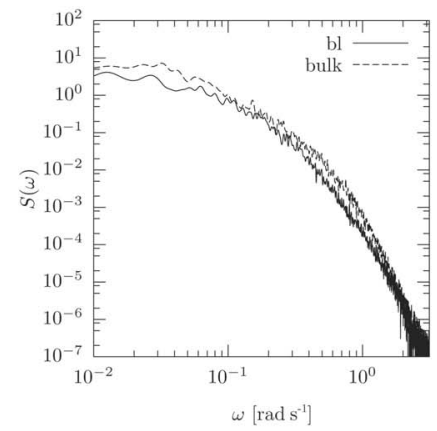

(c) $\mathrm{Ra}=2.6 \times 10^{6}$ (DNS)

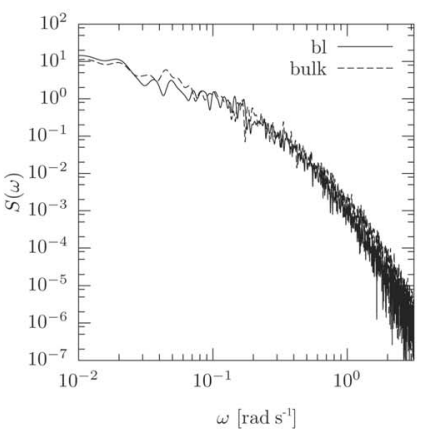

(g) $\mathrm{Ra}=7.7 \times 10^{7}(\mathrm{DNS})$

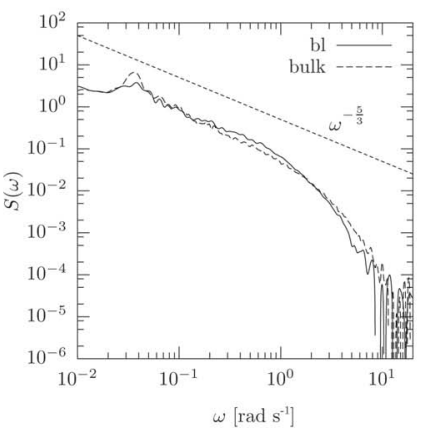

(j) $\mathrm{Ra}=2.4 \times 10^{6}(\mathrm{LDA})$

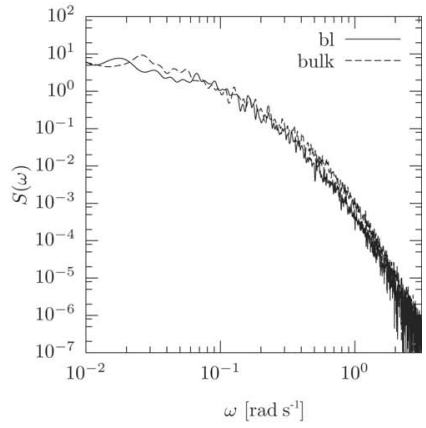

(d) $\mathrm{Ra}=7.2 \times 10^{6}$ (DNS)

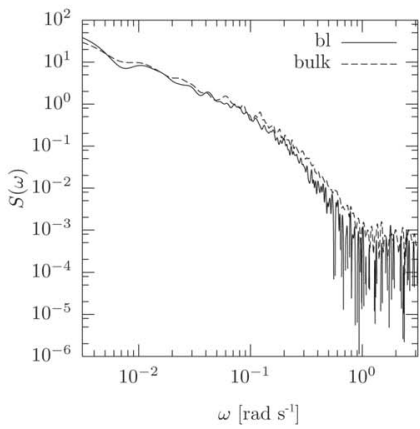

(h) $\mathrm{Ra}=8.2 \times 10^{7}$ (LDA)

FIG. 6. Spectral density functions for the horizontal velocity component $u$ for points in the boundary layer (bl) and in the bulk of the RB cell. Ra ranges from $7.0 \times 10^{4}$ to $2.4 \times 10^{9}$. The source of the data, DNS or LDA, is indicated.

in Fig. 6(b)] is dominated by a limited number of discrete peaks and the SDF rolls off rapidly at higher frequencies.

Peaks in the SDF also occur in the very low frequency range in Figs. 6(i) and 6(j). The peak frequencies $\omega_{\mathrm{p}}$ correspond to periods of $2 \pi / \omega_{\mathrm{p}}=283$ and $172 \mathrm{~s}$ at $\mathrm{Ra}=4.2 \times 10^{8}$ and $\mathrm{Ra}=2.4 \times 10^{9}$, respectively. These oscillations are unrelated to the ones observed at low $\mathrm{Ra}$, and have their origin in the alternating growth and decay of rolls that form the large scale circulation as discussed in [12].

For $\mathrm{Ra}>2.6 \times 10^{6}$ all SDFs measured in the bulk have a wide range of active scales, and this range increases with $\mathrm{Ra}$. These SDFs are continuous, and are not dominated by a small number of frequencies as one might expect in spatiotemporal chaos. Clearly these SDFs indicate that the bulk in the $\mathrm{RB}$ cell is turbulent for $\mathrm{Ra}>2.6 \times 10^{6}$. Although an inertial subrange is absent for the measurements at lower Ra, the $\mathrm{SDF}$ at $\mathrm{Ra}=2.4 \times 10^{9}[$ Fig. $6(\mathrm{j})]$ shows an inertial subrange spanning roughly one decade. Note that the presence of an inertial subrange is not a necessary condition for turbulence. An inertial subrange can only form when there is a range of scales which is influenced by neither macro- nor microscales, i.e., at high $\mathrm{Re}$ when the production and dissipation scales are sufficiently separated. Therefore an inertial subrange is absent despite the turbulence at lower Re (see also $[6,25])$.

The most striking observation is that the SDFs in the bulk and in the boundary layer are practically identical for $\mathrm{Ra}>2.6 \times 10^{6}$. As the SDFs for the bulk indicate that the flow is turbulent, one cannot escape from the conclusion that the flow in the boundary layers must also be characterized as turbulent. It is difficult to reconcile this observation with the conceptual image of a laminar, Blasius-type boundary layer along the walls of the RB cell. For a Blasius boundary layer, the laminar-turbulent transition occurs at a relatively high Reynolds number, i.e., $\operatorname{Re}_{c}^{*} \approx 10^{5}-10^{6}$ depending on the intensity and nature of disturbances in the flow [26]. Here, $\operatorname{Re}_{c}^{*}$ 
is a Reynolds number based on plate length and outer velocity. However, even at the highest Ra considered in this study, the value of $\mathrm{Re}^{*}$ based on the cell width and the wind velocity is only $3 \times 10^{3}$, which is far below the critical value for laminar-turbulent transition in a flat plate boundary layer. Thus the turbulence in the boundary layer cannot be due to a shear-triggered transition (occurring inside the boundary layer), but more likely due to the impingement and detachment of plumes (traveling into and out of the boundary layer), which is corroborated by the great similarity between the bulk and the boundary-layer spectra.

Naturally, one can always envision that the boundary layer only quantitatively behaves as a laminar Blasius boundary layer: the plumes introduce a time dependence on time scales that prevent pure laminarity. The underlying assumption is that on the average, the thermals and plumes are passive when it comes to scaling of various important integral parameters of the kinematic boundary layer such as the friction factor $C_{f}$ and the boundary layer thickness $\lambda_{u}$. Indeed, $C_{f}$ scales as $C_{f} \propto \mathrm{Re}^{-1 / 2}$, as one would expect for a laminar boundary layer past a flat plate at moderate $\mathrm{Ra}$ [9-11,27].

The scaling of the boundary layer thickness $\lambda_{u}$ is slightly more complicated. Assuming a laminar boundary layer, $\lambda_{u}$ scales as $\lambda_{u} \propto \mathrm{Re}^{-1 / 2}$ [26], which is related to $\mathrm{Ra}$ as $\lambda_{u} \propto \mathrm{Ra}^{-1 / 4}$ upon assuming $\mathrm{Re} \propto \mathrm{Ra}^{1 / 2}$. Experiments show that a scaling exponent -0.25 holds well for the boundary layer thickness of the sidewalls [28], but not for the scaling of the bottom and top boundary layers $[18,27,29]$ which scales with exponents from -0.11 to -0.16 . The simulations in this paper confirm the weak Ra number dependence of $\lambda_{u}$ (Fig. 2). This disparity indicates that plumes impinging on and emerging from the boundary layer are not entirely passive, but actively influence the boundary layer thickness. Given the important role of $\lambda_{u}$ in the theory of [8], it seems to us that a detailed study of how the interplay of plumes, the wind, and the boundary layer influences $\lambda_{u}$ is desirable. A first step in this direction is taken in a forthcoming study $[23,27]$, the results of which indicate that the scaling of $\lambda_{u}$ cannot be derived from laminar boundary layer theory and scales as $\lambda_{u} \propto \lambda_{\Theta}^{-1} \mathrm{Re}^{-1}$.

Although the SDFs point toward the presence of turbulence in the boundary layer, the characteristics of the boundary layer are of a rather different nature than those of classical (i.e., shear-driven) turbulent boundary layers. Indeed, viscous forces are still significant in the boundary layer, as can be deduced from the domain height $H$ in viscous units $\delta_{v}=\nu / u_{\tau}$, where $u_{\tau} \equiv \sqrt{\left.\nu \frac{\partial u}{\partial z}\right|_{\mathrm{W}}}$ is the friction velocity [26]. Assuming that $\left.\frac{\partial u}{\partial z}\right|_{\mathrm{w}} \approx U / \lambda_{u}$, the ratio $H / \delta_{v}$, which is the shear-Reynolds number $\operatorname{Re}_{\tau}$, can be approximated by

$$
\operatorname{Re}_{\tau} \equiv \frac{u_{\tau} H}{\nu} \approx\left(\frac{\lambda_{u}}{H}\right)^{-1 / 2} \operatorname{Re}^{1 / 2} .
$$

The simulations show that $\operatorname{Re}_{\tau}=70$ at $\operatorname{Ra}=2.1 \times 10^{7}$, and the viscous thickness of the boundary layer $\lambda_{u} / \delta_{v}=\lambda_{u} / H \operatorname{Re}_{\tau}$ is only a fraction of this already very low value. Therefore viscous effects are significant in the boundary layer.

It is interesting to note that the scaling of the boundary layer thickness for flat plate flow changes from $\delta / x \propto \mathrm{Re}_{x}^{-1 / 2}$ (laminar) to $\delta / x \propto \operatorname{Re}_{x}^{-1 / 7}$ (turbulent, see [30]), where $\operatorname{Re}_{x}$ is the Reynolds number based on the distance $x$ to the leading edge of the plate, and the thickness $\delta$ is the location where the velocity is $99 \%$ of the outer velocity. The decreased exponent resembles larger entrainment and thus a quicker growth of the boundary layer as a function of $x$. Upon assuming that the typical development length is $H$ and that $\operatorname{Re} \propto \mathrm{Ra}^{1 / 2}$, the anticipated scaling for a turbulent boundary layer is $\delta / H \propto \mathrm{Ra}^{-1 / 14}$. If $\alpha \approx-0.11$ is the scaling exponent $\lambda_{u} \propto \mathrm{Ra}^{\alpha}$ from the simulations, then $-1 / 4<\alpha<-1 / 14$, so the exponent may resemble an intermediate state.

However, it is not clear whether the entrainment mechanism of a developing boundary layer past a flat plate is transferable to RB convection; the active role of plumes which transfer fluid (and heat) into and out of the boundary layer may significantly affect the entrainment characteristics. It is our suspicion that the relatively weak forcing in the horizontal direction due to the wind (which is responsible for the friction factor $C_{f}$ ) is only weakly coupled to the relatively strong forcing in the wall-normal direction due to buoyancy. This may be the reason why the boundary layer has an SDF which is typical of developed turbulence but has many laminar features at the same time. Due to the weak coupling, the conceptual image of a laminar boundary layer may be appropriate for $C_{f}$ but not for $\lambda_{u}$ in the boundary layer in RB convection. Therefore the straightforward application of well-known characteristics of the Blasius-type boundary layer (such as the criteria for laminar-turbulent transition and the scaling of $\lambda_{u}$ with $\mathrm{Re}$ ) to the boundary layers in $\mathrm{RB}$ convection is questionable without additional justification.

\section{CONCLUSION}

A combined numerical and experimental investigation was carried out to study the boundary layers in RB convection in a 4:1 aspect-ratio cell filled with water. The study covers a wide range of $\mathrm{Ra}$ numbers varying between $7.0 \times 10^{4}$ and $2.4 \times 10^{9}$. The results for the lower Ra numbers were obtained from direct numerical simulations, while those for the higher Ra numbers followed from laser Doppler anemometry. In the midrange both techniques have an overlap of about one decade.

The probability density functions of the horizontal velocity components have a Gaussian-like shape for the complete Ra number range, except at the lowest Ra where the flow is in a state of spatiotemporal chaos. Probability density functions that are scaled with the velocity rms value overlap for the entire Ra number range, with the exception of the largest Ra numbers where the large-scale circulation, or "wind," is predominantly visible in the histograms.

The spectral density functions of the horizontal velocity component that were determined in the bulk flow and in the 
boundary layer are surprisingly similar. Spectra in both regions of the flow are continuous and have a wide range of active scales for $\mathrm{Ra}>2.6 \times 10^{6}$. This indicates that both the bulk and the boundary layers are turbulent in the Ra number range considered, while (i) molecular effects are still noticeable in the boundary layer and (ii) the boundary layer does not behave like a classical shear-driven turbulent boundary layer.
[1] E. D. Siggia, Annu. Rev. Fluid Mech. 26, 137 (1994).

[2] L. P. Kadanoff, Phys. Today 54 (8), 34 (2001).

[3] J. J. Niemela, L. Skrbek, K. R. Sreenivasan, and R. J. Donnelly, Nature (London) 404, 837 (2000).

[4] F. Heslot, B. Castaing, and A. Libchaber, Phys. Rev. A 36, 5870 (1987).

[5] B. Castaing, G. Gunaratne, F. Heslot, L. Kadanoff, A. Libchaber, S. Thomae, X.-Z. Wu, S. Zaleski, and G. Zanetti, J. Fluid Mech. 204, 1 (1989).

[6] X.-Z. Wu, L. P. Kadanoff, A. Libchaber, and M. Sano, Phys. Rev. Lett. 64, 2140 (1990).

[7] L. N. Howard, J. Fluid Mech. 17, 405 (1963).

[8] S. Grossmann and D. Lohse, J. Fluid Mech. 407, 27 (2000).

[9] X. Chavanne, F. Chillà, B. Castaing, B. Hébral, B. Chabaud, and J. Chaussy, Phys. Rev. Lett. 79, 3648 (1997).

[10] X. Chavanne, F. Chillà, B. Chabaud, B. Castaing, and B. Hébral, Phys. Fluids 13, 1300 (2001).

[11] G. Amati, K. Koal, F. Massaioli, K. R. Sreenivasan, and R. Verzicco, Phys. Fluids 17, 121701 (2005).

[12] J. Verdoold, M. J. Tummers, and K. Hanjalić, Phys. Rev. E 73, 056304 (2006).

[13] R. W. C. P. Verstappen and A. E. P. Veldman, J. Comput. Phys. 187, 343 (2003).

[14] M. van Reeuwijk, Ph.D. thesis, Delft University of Technology, Delft, 2007.
[15] K. Hanjalić, Int. J. Heat Fluid Flow 26, 828 (2005).

[16] S. Grossmann and D. Lohse, Phys. Rev. Lett. 86, 3316 (2001).

[17] X.-L. Qiu and P. Tong, Phys. Rev. E 64, 036304 (2001).

[18] R. M. Kerr, J. Fluid Mech. 310, 139 (1996).

[19] A. Vincent and M. Meneguzzi, J. Fluid Mech. 225, 1 (1991).

[20] H. Mouri, M. Takaoka, A. Hori, and Y. Kawashima, Phys. Rev. E 65, 056304 (2002).

[21] M. van Reeuwijk, H. J. J. Jonker, and K. Hanjalić, Phys. Fluids 17, 051704 (2005).

[22] J. J. Niemela and K. R. Sreenivasan, J. Fluid Mech. 557, 411 (2006).

[23] M. van Reeuwijk, H. J. J. Jonker, and K. Hanjalić, e-print arXiv:0709.0304.

[24] M. J. Tummers and D. M. Passchier, Meas. Sci. Technol. 12, 1641 (2001).

[25] R. Camussi and R. Verzicco, Phys. Fluids 10, 516 (1998).

[26] H. Schlichting and K. Gersten, Grenzschicht-Theorie, 9th ed. (Springer-Verlag, Berlin, 1997).

[27] M. van Reeuwijk, H. J. J. Jonker, and K. Hanjalić, e-print arXiv:0709.1891.

[28] X.-L. Qiu and K.-Q. Xia, Phys. Rev. E 58, 486 (1998).

[29] X.-L. Qiu and K.-Q. Xia, Phys. Rev. E 58, 5816 (1998).

[30] F. M. White, Viscous Fluid Flow (McGraw-Hill, New York, 1991). 